\title{
Efficient jamming signal against civilian GPS receivers
}

\section{Original Article}

\author{
Sherif A. Elgamel, Ehab M. Shaheen \\ Department of Electronic Warfare, Military Technical College, Cairo, Egypt
}

\section{Keywords:}

Civilian GPS receivers, GPS receiver tracking loop, jamming signal, loss of lock.

\section{Corresponding Author:}

Sherif A. Elgamel, Military Technical College, Cairo, Egypt, Tel: 2029666342

Email: elgamel@mtc.edu.eg

\section{Abstract}

Civilian Global Positioning System (GPS) receivers are subjected to different types of jamming/interference impacts, which degrade its accuracy and reliability.It is well known that GPS signals are considered weak signals; thus, it can be efficiently jammed by using a GPS jammer. One of the paper goals is to increase the interference effectiveness in such a way being able to jam multiple satellites simultaneously, causing continues loss of lock for the GPS receiver tracking loop and decreasing the mitigation probability; a novel jamming signal is proposed which increases the interference influence zone and affects more than one satellite simultaneously. The proposed jamming signal has the ability to generate continuous loss of lock for the GPS receiver-tracking loop and to decrease the mitigation probability. The proposed jamming signal affects all GPS satellites with nearly small interference tolerance value and causes continuous loss of lock to the carrier-tracking loop of the GPS receiver.

\section{INTRODUCTION}

The GPS is a satellite navigation system, which gives peopl earound the globe the abilities of navigation accuracy and perfect timing.

Nowadays, GPS systems with a reasonable moderate price can provide very attractive results that could reach centimetres range of accuracy.

Thus, the GPS accuracy is not considered an outstanding challenge from the point of view of GPS receiver designers, yet, focusing on enhancing the device's robustness. One of the main problems that could deteriorate the efficiency of the GPS system is the presence of in band high power interference or jamming signals.

In the literatures, many references talked about the antijamming techniques that can be used to insure the GPS works in the presence of jamming signals ${ }^{[1-5]}$, but very little references regarding GPS jamming. $\mathrm{In}^{[6]}$, a presentation to a novel classification of different interference signals was depicted. Theidea of this new classification was based on both the coherentintegration time of the GPS correlator and the bandwidth of the interference signal. This new classification provided simplified analytical closed formulas for the GPScorrelator output power where many types of jamming/interference signals are present as: narrowband interference $(\mathrm{NBI})$, continuous wave interference $(\mathrm{CWI})$, broadband interference (BBI) and partial band interference (PBI). In ${ }^{[7]}$, novel analytical formulas were derived with these mentioned types of jamming signals are present. Their impact on the tolerance of the GPS receiver along with the mean time to loss lockwere investigated.
Thus, the objectives of this workcan be statedas:proposing an optimum interference signal, this affects all satellites with nearly small interference tolerance value and causes continuous loss of lock in the GPS carriertracking loop.

This paper is organized as follows: the GPS receiver structure and jamming signals are summarized in section (2). In section (3), the Interference effect on GPS carrier to noise ratio $\left(C / N_{o}\right)$, acquisition process and tracking loops are presented. The proposed optimum GPS jamming signal is presented in section (4). Section (5) draws the conclusions of the paper.

\section{GPS RECEIVER AND JAMMING SIGNALS}

GPS systems actually operates on two major frequencies, the first one is dedicated for civilian use $(1575.42 \mathrm{MHz})$ whereas the other is considered for military applications (1227.6 MHz). GPS signals are vulnerable signals; which can be jammed or distorted, thusmany efforts have to be donein order to mitigate the jammer's impact on the GPS systems. GPS jammers were initially presented by governments, for military applications to confuse the enemy GPS guided missiles or bombs in certain locations.

\section{II.1. GPS Receiver}

The GPS receiver has to accomplish several different functions, from the acquisition and tracking of the signals transmitted by satellite to the computation of the user's position. Fig.1. displays the main block diagram of a GPS receiver. 


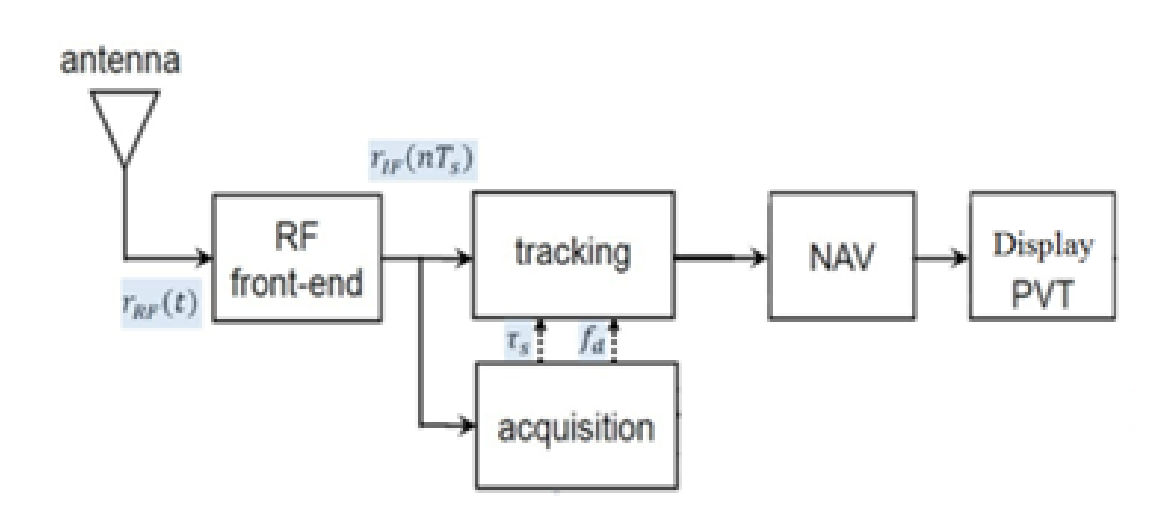

Fig. 1: GPS receiver's block diagram.

The signal at the input of the GPS receiver is $160 \mathrm{dBw}$ at the antenna input, spread over about $2 \mathrm{MHz}$ bandwidth by the spread spectrum GPS L1 coarse acquisition code (C/A), most of the power can be found in the central 2 MHz section ${ }^{[8]}$.

The GPS receiver input signal can be modelled as:

$$
\begin{aligned}
r_{R F}(t)=\sum_{i=1}^{V} \sum_{i=1}^{3} & \sqrt{2 \cdot P_{r_{i j}}} D\left(t-\tau_{s_{j}}\right) C_{i}(t \\
& \left.-\tau_{s_{j}}\right) \cdot \cos \left[2 \pi\left(f_{R F_{i}}+f_{d_{i j}}\right) t\right. \\
& \left.+\varphi_{i}+\theta_{i j}\right]+I(t)+\mu(t)
\end{aligned}
$$

Where $\mathrm{P}_{r i j}$ is the received signal power, $\tau_{s j}$ is the time delay introduced of the $\mathrm{j}$-the satellite, $\mathrm{f}_{d i j}$ is the Doppler frequency shift, and $\theta_{i j}$ is the carrier phase introduced by the channel. The receiver is able to process each signal component independently. $\varphi_{i}$ is the phase term used to indicate if the signal component has been broadcast inphase or quadrature, $I(t)$ is the interference signal, and $\mu(t)$ is the Additive White Gaussian Noise (AWGN), $D(t)$ is the navigation message, containing the GPS time, the satellite health status and the almanacs, $\mathrm{C}(\mathrm{t})$ is the spectrum of the periodic $\mathrm{C} / \mathrm{A}$ code , and $\mathrm{V}$ is the number of satellites in view.

In this paper, the $\mathrm{L} 1-\mathrm{C} / \mathrm{A}(\mathrm{C} / \mathrm{A}$ code signal at frequency L1) signal is studied while the other signals (L1-P(Y), L2-P(Y)) are ignored, because the lack of the $\mathrm{P}(\mathrm{Y})$ code references. Thus, the index is removed in the following equation. The received signal can be rewritten as:

$$
\begin{aligned}
r_{R F}(t)=\sum_{i=1}^{V} \sqrt{2 . P_{r j}} D_{j}\left(t-\tau_{s_{j}}\right) C(t \\
\left.-\tau_{s_{j}}\right) \cdot \cos \left(2 \pi\left(f_{R F}+f_{d_{j}}\right) t+\theta_{j}\right. \\
\left.+90^{\circ}\right)+I(t)+\mu(t)
\end{aligned}
$$

\section{II.2. GPS Jamming Signals}

Interference can be generally defined as any undesired signal that disturb the proper operation of the GPS system.
Interference signal is a major source for degradation of the GPS accuracy and reliability. The GPS frequency bands are internationally protected by the Federal Communication Commission frequency assignments ${ }^{[9]}$. Unintentional interference can be caused by inband operating services or other accidental transmission of signals in the dedicated GPS band. The interference can also be caused by jamming signal transmitted from intentional jammers ${ }^{[10]}$. The more complex jamming techniquesare the repeater and spoofer techniques ${ }^{[11,12]}$.

The main strategy for any interference mitigation method is to eliminate and reduce the interference impact on the performance of the GPS system. The main mitigation methods are: RF filtering ${ }^{[10]}$, Pulse blanking ${ }^{[13,14]}$, Notch filtering,adaptive antenna array ${ }^{[15]}$, andautomatic gain control as an interference mitigation tool ${ }^{[16]}$.

\section{JAMMING EFFECT ON GPS $C / N_{o}$, ACQUISITION PROCESS AND TRACKING LOOPS}

In this section, an accurate definition for both matched spectrum interference(MSI) andCWI are introduced also the interference impact on the $C / N_{o}$, acquisition process and tracking loops will be investigated.

\section{III.1. CWI and MSI Effect at the Correlator Output}

It worth to be noted that the frequency resolution in the correlator equals $1 / T_{d}$. Thus, one can consider that we have a CWI interference/jamming type signal if $\left(B_{N} \leq \frac{1}{T_{d}}\right)$, where $\mathrm{BN}$ is the interference bandwidth. Noted that the GPS receive frontend uses an IQ sampling method to provide GPS correlator input with the baseband complex signal. For that it can be written as:

$$
I_{C W}\left(n T_{s}\right)=\sqrt{P_{j}} \cdot e^{j\left(2 \pi f_{j} n T_{s}+\theta_{j}\right)}
$$

where $\mathrm{P}_{j}$ is the power of the interference signal, $f j$ is the frequency of the CWI signal at the correlator input, and $\theta j$ is the phase of the jamming/interference signal.

Thus in presence of the CWI signal: 


$$
\begin{aligned}
P_{C W}\left(\tau_{n}, \Delta f_{j}\right)=K & \mid \frac{1}{N_{s}} \sum_{n=0}^{N_{s}-1} \sqrt{P_{j}} C\left(n T_{s}\right. \\
& \left.-\tau_{n}\right)\left.e^{j\left(2 \pi \Delta f_{j} n T_{s}+\Delta \theta_{j}\right)}\right|^{2}
\end{aligned}
$$

Where $\mathrm{K}$ is the non-coherent integration time, the error in frequency for interference/jamming signals, $\Delta f_{j}$ is the difference between the jamming frequencies, $f_{j}$ and the reference carrier frequency of the receiver $\left(\Delta f_{j}=f_{j}-\hat{f}_{D}\right)$, and $\Delta \theta_{j}$ is the phase error. Fig. 2. displays spectral lines representation of the CWI and the GPS reference signals.

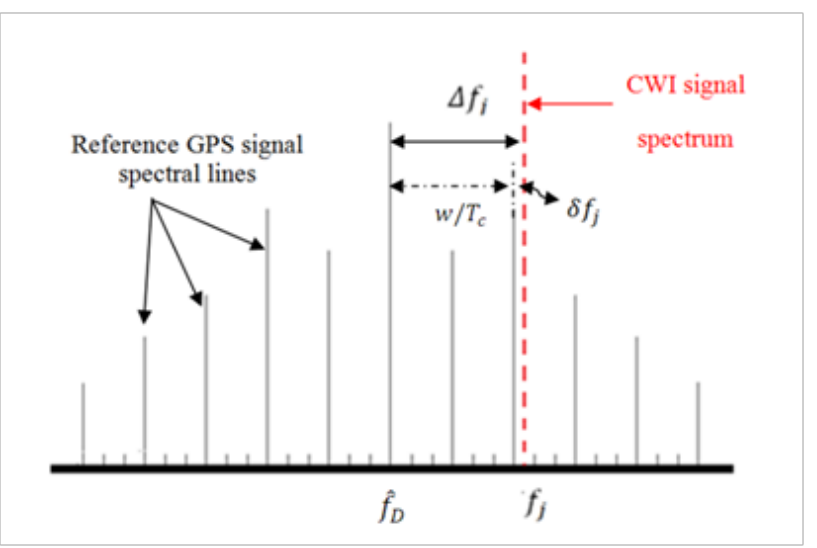

Fig. 2: Reference GPS and CWI signals spectral lines.

From Fig. 2 it can be seen thatthe reference signal has a spectrum similar to the C/A code spectrum modulated by reference frequency $f_{D}^{\wedge}$.

The MSI signal can be described as that kind of interference/jamming that resemblesexactly the GPS modulation, signal code, spectral characteristics and chipping rate ${ }^{[17]}$.

In this section, the effect of the cross correlation sequence (CCS) spectral lines in the MSI effect is investigated. Fig. 3 shows the spectral lines of both the reference GPS and MSI signals.

One can model the matched spectrum interference at the correlator's in put as:

$$
I_{M S}\left(n T_{s}\right)=\sqrt{P_{j}} \cdot P\left(n T_{s}-\tau_{p}\right) \cdot e^{j\left(2 \pi f_{j} n T_{s}+\theta_{j}\right)}
$$

Where $P_{j}$ is the interference/jamming power, $P\left(n T_{s}\right)$ is the spreading code of the MSI C/A, $\tau_{p}$ is the code delay of the MSI, $f_{j}$ and $\theta_{j}$ are the jamming frequency and phase, respectively.

The GPS correlator output power is investigated in presence of this kind of interference signal $\operatorname{asr}\left(n T_{s}\right)=I_{M S}\left(n T_{s}\right)$.

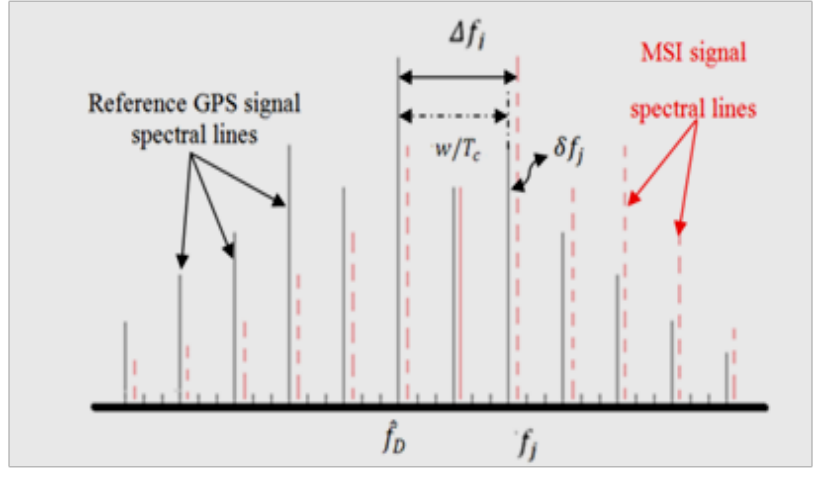

Fig. 3: GPS and MSI signals spectral lines.

MSI interference/jamming power at the correlator output is modelled as:

$$
\begin{aligned}
P_{M S}\left(\Delta \tau_{p}, \Delta f_{j}\right)= & K \mid \frac{1}{N_{s}} \sum_{n=0}^{N_{s}-1} \sqrt{P_{j}} . P\left(n T_{s}\right) C\left(n T_{s}\right. \\
& \left.+\Delta \tau_{p}\right)\left.e^{j\left(2 \pi \Delta f_{j} n T_{s}+\Delta \theta_{j}\right)}\right|^{2}
\end{aligned}
$$

Where $\Delta \tau_{p}$ is the code phase error of the MSI $\left(\Delta \tau_{p}=\tau_{\mathrm{s}}-\tau_{\mathrm{p}}\right)$. The periodic $P\left(n T_{s}\right)$ code can be modelled as:

$$
P\left(n T_{s}\right)=\sum_{i=-N_{C}}^{N_{c}} p_{i} e^{j 2 \pi \frac{i}{T_{c}} n T_{s}}
$$

Where $p i$ are the Fourier series coefficients of $P\left(n T_{s}\right)$. One can model the impact of the MSI jamming at the GPS correlator output power as (see (8))

Consider $m=i+q$, then the GPS correlator output power with the existence of MSI jamming signal is (see (9)).

\section{III.2 CWI and MSI Impact on the GPS Correlator Output Power}

Noted that with the existence of CWI, its effect on the output power of the GPS correlator depends on the amplitude of the GPS code. Also,when multiple satellites transmit GPS signal which in turn was subjected to the same CWI signal, the GPS signal will be affected differently to this interference signal. It is found that the CWI signal will cause the greatest impact (minimum $C / N_{o}$ value) on the GPS system, when this interference/jamming is being at a specified offset with the carrier frequencyof the GPS signal (42 kHz and $263 \mathrm{kHz}$ for PRN1 and PRN2 respectively). The CWI degrades the $C / N_{o}$ value when the 
difference between the nearest spectral line of the C/A code and the reciprocal of the integration timeis greater thanthe interference frequency. This is because the width of the $C / N$ Since trough around each spectral line equals to $\frac{2}{T_{d}} \quad[6,18]$.

When the interference/jamming frequency equals to the received frequency of the GPS its impact will be smaller than the scenario whenthe CWI frequency is not identical with the GPS carrier frequency. Such scenariooccurs as the amplitude of the spectral line of the C/A code number zero is too small when compared with the neighbour spectral line.

A drop in the $C / N_{o}$ value occurs when the matched spectrum interference gets over the GPS signal spectrallines.

This drop depends on the CCS spectral-line'samplitude. The MSI influence zone related to integration time, and the $C / N_{o}$ value decrease by increasing the integration time.

\section{III.3 GPS Acquisition Process Performance in Presence of CWI and MSI}

Clear large peaks is noticed in presence of two types of interference/jamming signals (MSI and CWI), when the $\mathrm{C} / \mathrm{A}$ code periodis less than the interference/jamming signal pulse width or when the code chip durationis greater than the interference pulse period.

It was proved that in ${ }^{[20]}$ these two interference/jamming signals gave contribution to the correlator output mean value.

It can be seen in Fig 4. the GPS acquisition output in absence of interference. Fig. 5. depicts the impact of presence of CWI at the acquisition output at different time integration values. Fig. 6., Fig. 7 and Fig. 8. present the impact of presence of PBI, BBI and MSI, respectively.

One can notice that depending on the phase relation between the interference and GPS signal components, these two signals (GPS and jamming) can be either added or subtracted. In this scenario, the correlator output variance is assumed equals to the noise variance. Interference/ jamming signals increase the noise level at the output of the GPS acquisition process, and their contributions increase the variance valueof the GPS correlator output.

Also, the amplitude of the GPS signal affects the output mean value of the GPS correlator, where both MSI and CWI jamming/interference signals gave the greatest impact on the GPS acquisition process, then the narrow band interference impact followed by the pulse interference impact. Finally, the weakest impact occurs in presence of either PBI or BBI ${ }^{[6,18,20]}$. It was shown that decreasing the integration timewill lead to a decrease in the performance of the GPS acquisition process ${ }^{[6,18,20]}$.

$$
\begin{gathered}
P_{M S}\left(\Delta \tau_{p}, \Delta f_{j}\right)=K \cdot P_{j}\left|\frac{1}{N_{s}} \sum_{n=0}^{N_{s}-1} \sum_{i=-N_{c}}^{N_{c}} p_{i} e^{j 2 \pi \frac{i}{T_{c}} n T_{s}} \cdot \sum_{q=-N_{c}}^{N_{c}} c_{q} e^{j 2 \pi \frac{q}{T_{c}} n T_{s}} e^{j 2 \pi \frac{\Delta \tau_{p}}{T_{c} q}} \cdot e^{j\left(2 \pi \Delta f_{j} n T_{s}+\Delta \theta_{j}\right)}\right|^{2 !} \\
=K \cdot P_{j}\left|\frac{1}{N_{s}} \sum_{n=0}^{N_{s}-1} \sum_{i=-N_{c}}^{N_{c}} \sum_{q=-N_{c}}^{N_{c}} p_{i} c_{q} e^{j 2 \pi \frac{\Delta \tau_{p}}{T_{c} q}} e^{j\left(2 \pi\left(\frac{i+q}{T_{s}}+\Delta f_{j}\right) n T_{s}+\Delta \theta_{j}\right)}\right|^{2 !} \\
P_{M S}\left(\Delta \tau_{p}, \Delta f_{j}\right)=K \cdot P_{j}\left|\sum_{m=-N_{c}+q}^{N_{c}+q} \sum_{q=-N_{c}}^{N_{c}}\left(p_{m-q} c_{q} e^{j 2 \pi \frac{\Delta \tau_{p}}{T_{c}} q}\right) \cdot \frac{1}{N_{s}} \sum_{n=0}^{N_{s}-1} e^{j\left(2 \pi\left[\frac{m}{T_{c}}+\Delta f_{j}\right] n T_{s}\right)}\right|^{2}
\end{gathered}
$$

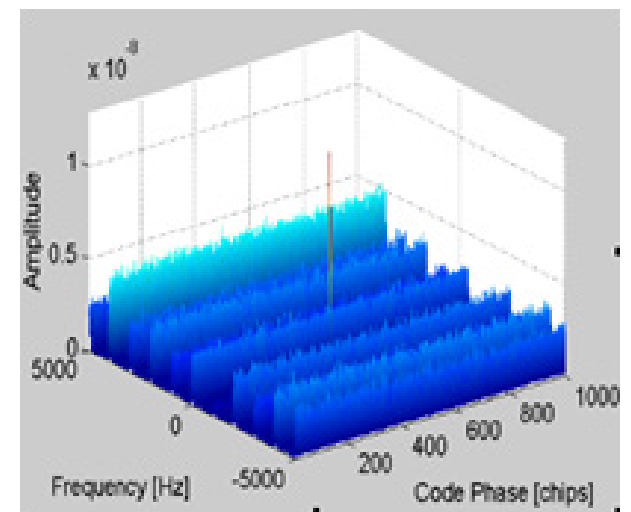

(a)

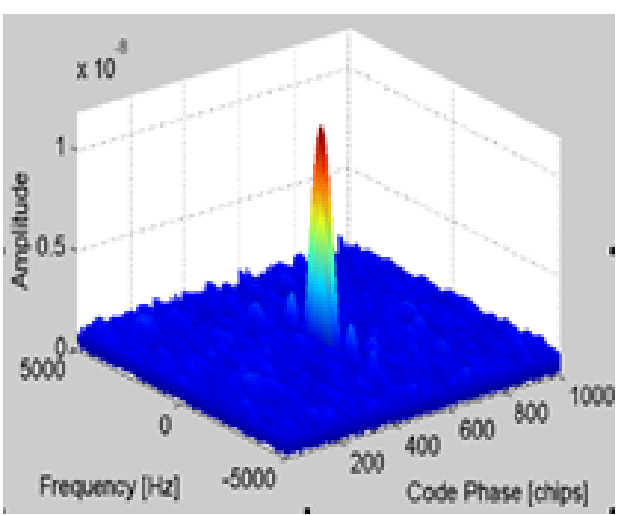

(b)

Fig. 4: The acquisition output for the GPS signal in the absence of interference. a:Acquisition output $\left(\mathrm{T}_{\mathrm{d}}=20 \mathrm{~ms}\right)$; b: Acquisition output $\left(\mathrm{T}_{\mathrm{d}}=5 \mathrm{~ms}\right)$ 


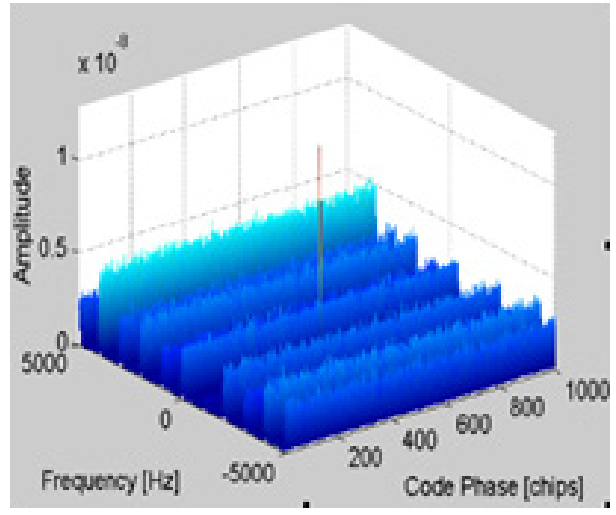

(a)

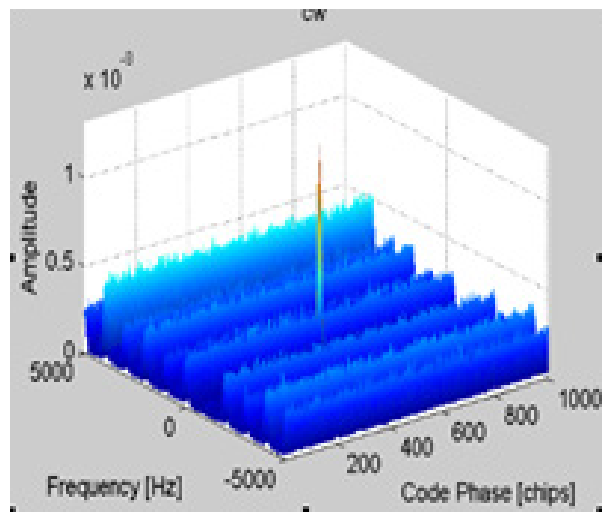

(b)

Fig. 5: The acquisition output for the GPS signal in the presence of CWIat different integration time values. a: Acquisition output $\left(\mathrm{T}_{\mathrm{d}}=20 \mathrm{~ms}\right), \mathrm{b}$ :Acquisition output $\left(\mathrm{T}_{\mathrm{d}}=5 \mathrm{~ms}\right)$.

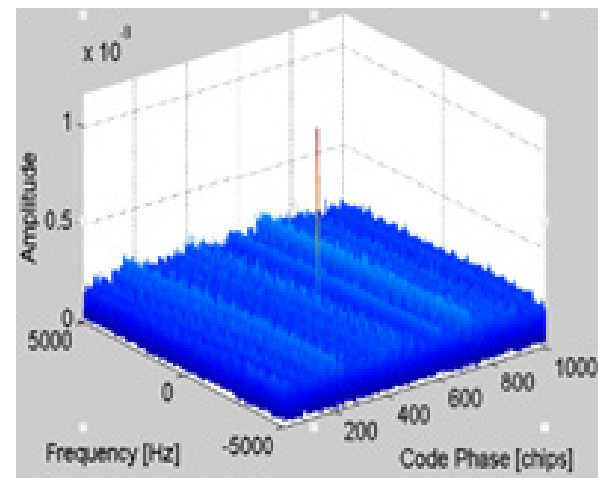

(a)

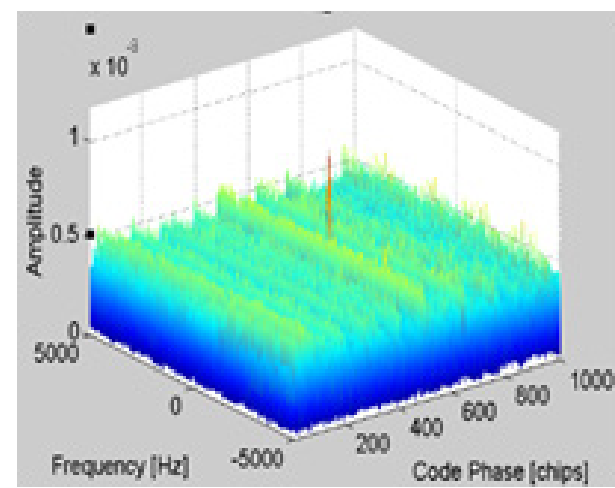

(b)

Fig. 6: The acquisition output for the GPS signal in the presence of PBI. a:Acquisition output $\left(\mathrm{T}_{\mathrm{d}}=20 \mathrm{~ms}\right)$, b:Acquisition output $\left(\mathrm{T}_{\mathrm{d}}=5 \mathrm{~ms}\right)$

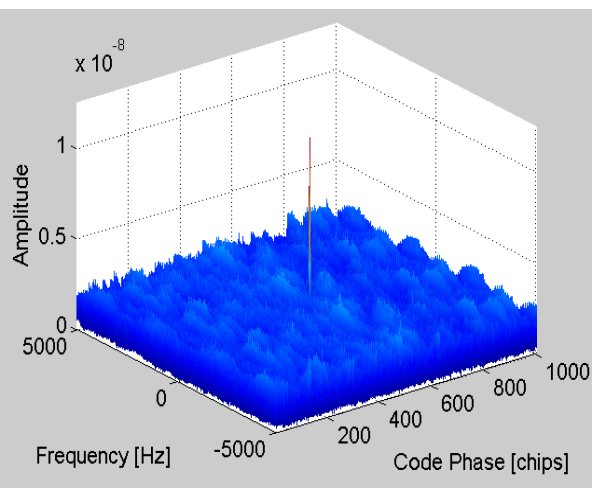

(a)

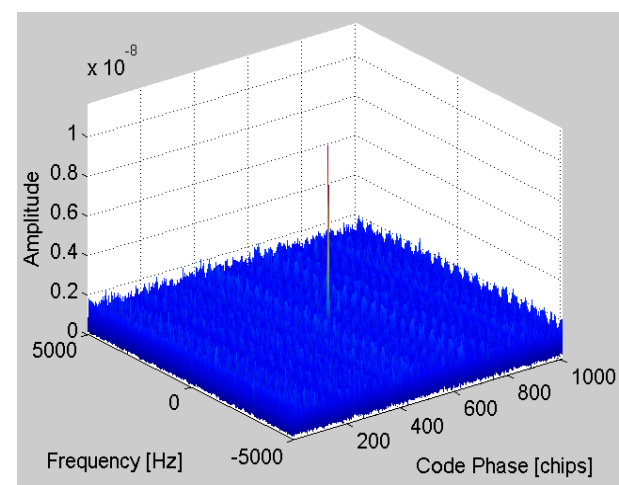

(b)

Fig. 7: The acquisition output for the GPS signal in the presence of BBI at different bandwidth values, a: Acquisition output $\left(\mathrm{B}_{\mathrm{N}}=3 \mathrm{MHz}\right), \mathrm{b}$ : Acquisition output $\left(\mathrm{B}_{\mathrm{N}}=6 \mathrm{MHz}\right)$ 


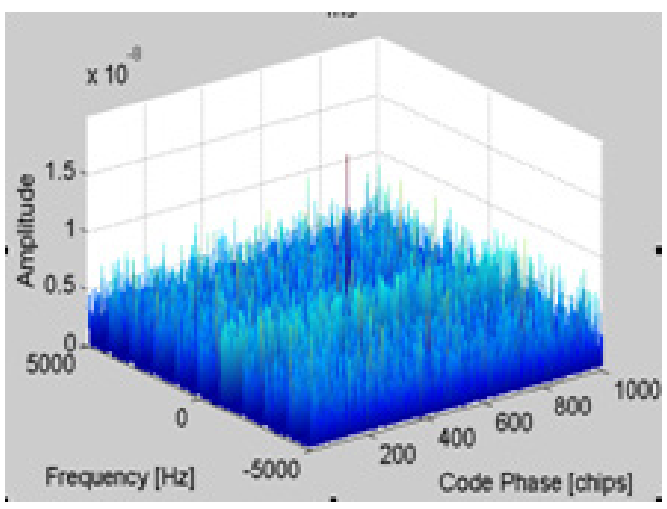

(a)

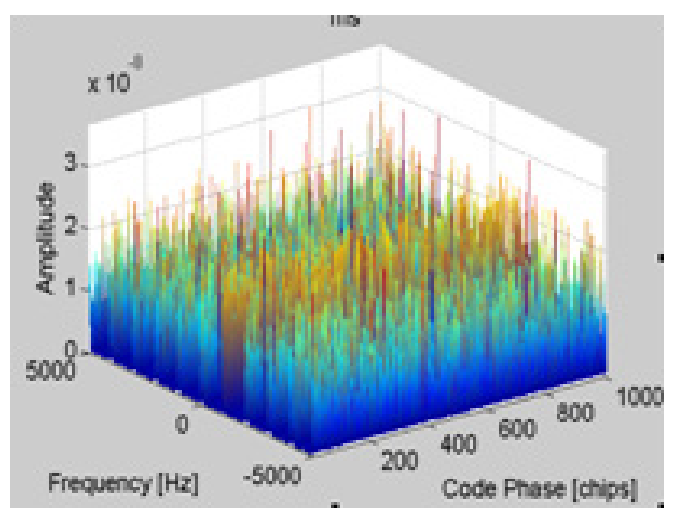

(b)

Fig. 8: The acquisition output for the GPS signal in the presence of MSI at different power level values, a:Acquisition output $\left(\frac{P_{j}}{P_{s}}=20 d B\right)$, b:Acquisition output $\left(\frac{p_{j}}{p_{s}}=30 d B\right)$

\section{III.4 GPS Tacking Loop Performance with CWI and MSI}

It was proven that ${ }^{[6,18]}$ when $1 / T_{d}$ was greater than the difference between the frequency of the interferer and the closest C/A spectral line; continues wave interference signal gave a significant impact on the receiverof the GPS system.

In our work, in order to jam more than one satellite in the same time, one mustincrease the interference influence zone, causing continues GPS tracking loop lock loss and decreasing the mitigation probability; a new interference signal is proposed. The proposed interference signal is a sweeping combination of MSI and CWI. The sweeping parameters are defined as follows: the sweeping step should be less than or equal to $1 / T_{d}$, the sweeping frequency range width should be grater or equal to $1 \mathrm{kHz}$, the sweeping, dwell time at each sweep frequency should be longer than the MTLL value and the sweeping speed should be adjusted to guarantee that the interference frequency returns to its influence zone for time less than the reacquisition time.

\section{THE PROPOSED OPTIMUM GPS JAMMING SIGNAL}

$\operatorname{In}^{[6,18,7]}$, the interference effect on the $C / N$, acquisition process and tracking loops was investigated. One can conclude that both CWI and MSI have the largest interference impact on the GPS system. It was noted that these two interference signals have their highest impact when two conditions were fulfilled. When $1 / T_{d}$ is greater than the frequency resolution of the CWI signal and the nearest GPS spectral line, and once the MSI spectral-lines are on the GPS spectral-lines with difference less than the $1 / T_{d}$ too. In these cases, the CWI and MSI with low jamming power levels will have a very noticeable effect and cause a GPS lock loss and vice-versa.

It should be noted that the relative motion of the GPS satellite to the GPS system is responsible a Doppler shift ranges- $5 \mathrm{kHz}$ and $+5 \mathrm{kHz}$. The average rate of change is $\delta f_{d r} \approx 0.54 \mathrm{~Hz} / \sec ^{[19]}$. Thus, with a fixed jamming frequency, it will catch only temporarily the GPS spectral line (especially when the integration timevalue is very long, as in such case the CWI and MSI influence zone become very narrow).

Generally, for a fixed interference frequency (MSI/CWI) only one satellite at a time is jammed due to Doppler shift effect. In the scenario when more than four satellites are existed to provide the position calculation, the GPS receiver can acquire its position using an optimization technique. For that it can be concluded that, the fixed interference frequency (CWI/MSI) can't prohibit the GPS system from doing its job, except in the case that the CWI or MSI has a very high power at the GPS receiver input.

\section{IV.1 The Proposed Interference Signal Characteristic}

In order to efficiently jam more than one satellite simultaneously, one mustincrease the interference influence zone, the sweeping CWI or sweeping MSI signal is proposed. These types of interference signals can capture all the received satellite signals by sweeping over the C/A code and CCS spectral lines.

If $\left.\left(\Delta \tau_{p}=\mathrm{nT}\right)_{a}\right)$, where $\Delta \tau_{p}$ is the code error in presence of MSI signal, and $\mathrm{T}_{a} \mathrm{C} / \mathrm{A}$ code chip duration, in this scenario the effect of the MSI signal can be generally considered equivalent to the effect of the CWI, otherwise the MSI effect will be decreased (the MSI effect decreases to half when $\left.\Delta \tau_{p}=1 / 2 \mathrm{~T}_{a}+\mathrm{nT}_{a}\right)$.

It is worth to be noted that one of the disadvantages of the MSI signal is the $\Delta \tau_{p}$ value. This value is related to the reference code delay and is unknown and hard to be determined. However, the MSI has the same GPS C/A code, BPSK as a modulation technique, and the $1.023 \mathrm{MHz}$ chip rate. Thus, it can be considered hasthe GPS signal spectral characteristics and it can be considered as the most difficult interference waveform for the GPS receivers to mitigate.

Also, for the CWI case, since the GPS signal is a spread spectrum signal; a narrow band filter can remove the CWI signal along with a small portion of the GPS 
signal will be removed. To this end;in order to decrease the mitigation probability, the proposed interference signal is a combination of MSI and CWI.

The key parameters in designing the sweeping interference are: the sweeping frequency range $\Delta f_{w}$, the sweeping frequency step $\Delta f$, and the dwell time at each frequency step $T_{D}$. Depending on these sweeping parameters, the proposed interference signal will be able to make a continues GPS tracking loop lock loss even though its frequency moves away from the influence zone.

To determine these sweeping parameters, the value of the receiver coherent integrating time, the carrier tracking loop mean time to loss lock (MTLL) and the reacquisition time are required. The GPS receiver can remain in unlock state if the following conditions are achieved:

As the frequency interval between any two spectral line equals to $1 \mathrm{kHz}$, thus the sweeping frequency range width should be greater or equal to $1 \mathrm{kHz}$. To insure that, there is at least one spectral line within the sweeping range and the proposed interference signal will be able to affect at least one spectral line at a specific time.

The condition $\left(\delta f_{j}<1 / \mathrm{T}_{d}\right)$ should be achieved for time longer than the MTLL value in order to increase the probability of loss lock. Thus, the sweeping dwell time at each sweep frequency should be longer than the MTLL value.

The GPS receiver takes time before the reacquisition process and recovers from the unlock state, that time is called the reacquisition time. Thus, the sweeping speed should be adjusted to guarantee that the interference frequency returns to its influence zone for time less than the reacquisition time

IV.2 Proposed Jamming Signal Generation and Analysis

One can model the proposed jamming signal as:

$$
\begin{aligned}
I_{P I}\left(n T_{s}\right)=\sqrt{P_{j} / 2} \cdot & {\left[P \left(n T_{s}\right.\right.} \\
& \left.-\tau_{p}\right) \cdot e^{j\left(2 \pi\left(f_{j}+v \cdot \Delta f_{s}\right) n T_{s}+\theta_{j}\right)} \\
& \left.+e^{j\left(2 \pi\left(f_{j}+v \cdot \Delta f_{s}\right) n T_{s}+\theta_{j}\right)}\right]
\end{aligned}
$$

Where $\Delta \mathrm{f}_{\mathrm{s}}$ is the sweeping step, $\Delta f_{w}$ is the sweeping frequency range, the number of the interference step is equal to $\mathrm{N}_{\mathrm{e}}=\frac{\Delta f_{w}}{\Delta f}$. Thus, $\mathrm{v}$ has a value changes from 0

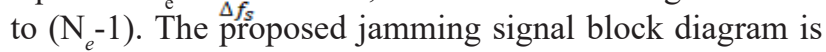
displayed in Fig. 9.

The sweeping control needs the sweeping range and the dwell time values to calculate the sweeping step and the $\mathrm{v}$ values which applied to the Sweep CWI generator (SCWIG). The SCWIG output divided into two branches, one of them multiply by the C/A code to generate the sweep
The reference GPS and proposed interference signal spectrum are shown inFig. 10. It can be noted that, the proposed interference signal is a combination of the SMSI and SCWI signals with interference frequency error $\Delta \mathrm{f}_{\mathrm{j}}$.

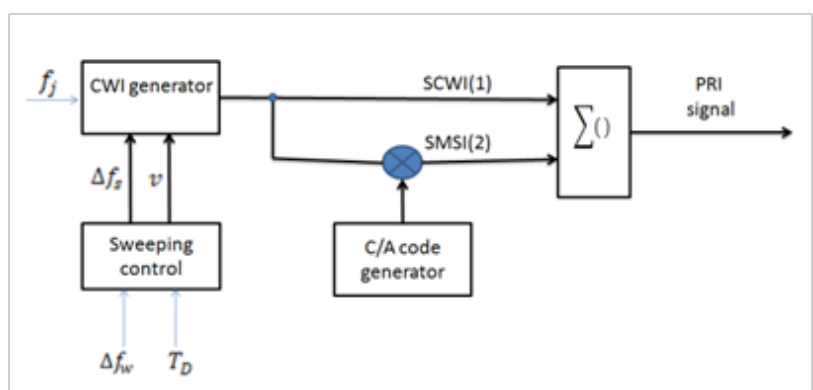

Fig. 9: Block diagram of the proposed interference signal generator.

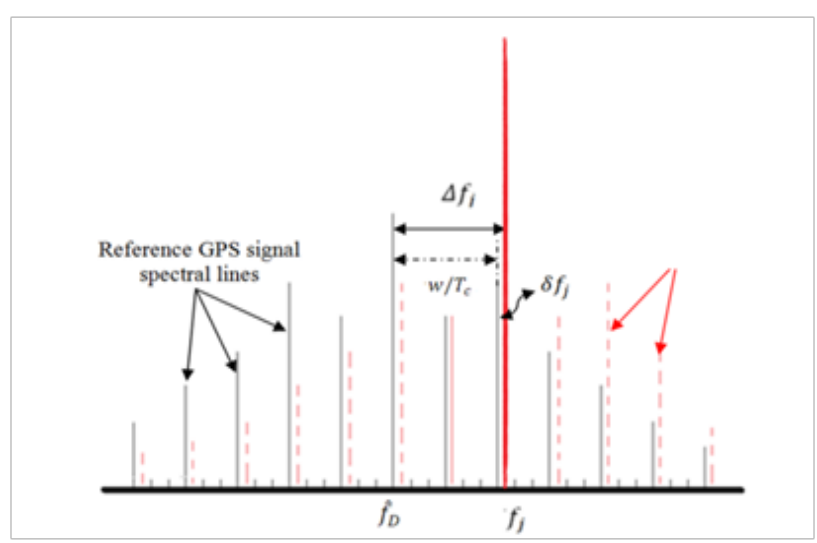

Fig. 10: Reference GPS and proposed interference signal spectral lines.

The output power of the proposed jamming signal can be written as:

$$
\begin{aligned}
& P_{P I}(v) \\
& =K \cdot P_{j}\left(\frac{\left|\grave{g}_{w(v)}\right|^{2}+\left|\grave{c}_{w(v)}\right|^{2}}{2}\right)\left(\operatorname{sinc}\left(\pi\left(\delta f_{j}(v)\right) T_{d}\right)\right)^{2}
\end{aligned}
$$

Where the interference frequency error equals to

$$
\Delta f_{j}=\left(f_{j}+v \cdot \Delta f_{s}\right)-\hat{f}_{D}=\frac{w(v)}{T_{c}}+\delta f_{j}(v)
$$

MSI (SMSI). Finally, the SCWI and SMSI is summed to generate the proposed interference signal (PRI).

As each satellite has a different Doppler frequency and different $\mathrm{C} / \mathrm{A}$ code, thus, the proposed jamming signal will be coincident with different spectral lines and each one has different amplitude. From the C/A code spectrum it is noted that the first 300 spectral line contains the largest amount of the $\mathrm{C} / \mathrm{A}$ code power. From extensive simulation 
experiments, the mean value of the amplitude for the first 300 spectral line is nearly equal to 0.027 and the mean value of the CCS spectral line amplitude at different $\Delta \tau_{\mathrm{p}}$ value is nearly equal to 0.02 .

Substitute $\left|\grave{\mathrm{g}}_{\mathrm{w}(\mathrm{v})}\right|=0.02$ and $\left|\grave{\mathrm{c}}_{\mathrm{w}(\mathrm{v})}\right|=0.027 \mathrm{in}(11)$, then

$$
P_{P I}(v)=(0.00056) K \cdot P_{j}\left(\operatorname{sinc}\left(\pi\left(\delta f_{j}(v)\right) T_{d}\right)\right)^{2}
$$

As the proposed interference signal sweeping step is considered to be less than or equal to1/T_d; the proposed interference signal coincident with a spectral line with error less than $1 / \mathrm{T}_{-} \mathrm{d}$, then the term $\left(\sin c\left(\pi\left(\delta f_{j}(v)\right) \mathrm{T}_{d}\right)\right)$ can be considered equals to one.

Then:

$$
P_{P I}(v)=(0.00056) K . P_{j}
$$

Finally, the proposed interference signal tolerance can be written as:

$$
K_{P I S}=\frac{P_{j_{-} a n t}}{P_{s_{-} a n t}}=\frac{L_{M} \cdot G_{s} \cdot \gamma\left(\operatorname{sinc}\left(\pi \Delta f_{D} T_{d}\right)\right)^{2}}{(0.00056) G_{j} \cdot B_{n} \sqrt{B_{n}^{2}+\frac{\gamma B_{n}}{T_{d}}} \cdot T_{d}}
$$

When $\mathrm{N}_{\mathrm{o}}=-203 \mathrm{dBW} / \mathrm{Hz}, \mathrm{T}_{d}=20 \mathrm{~ms}, \mathrm{~B}_{\mathrm{n}}=5 \mathrm{~Hz}, \mathrm{G}_{\mathrm{s}}=1$, $\mathrm{L}_{\mathrm{i}}=1, \mathrm{~L}_{\mathrm{M}}=1$ and $\mathrm{G}_{\mathrm{j}}=1$, then the proposed interference tolerance $\mathrm{K}_{\mathrm{PIS}}=28.8 \mathrm{~dB}$. This value is more than the continues wave interference tolerance when its frequency is onGPS code worst line. Yet, the proposed jamming signal effects all the satellites with nearly small jamming tolerance value and causes a continues lock loss when compared with the fixed frequency CWI or MSI cases which effect only one satellite temporarily.

\section{CONCLUSION}

In this paper, in order to jam efficiently more than one satellite simultaneously, we have to increase the interference influence zone, causing continues GPS tracking loop lock loss and decreasing the mitigation probability; a new interference signal was proposed. The proposed interference signal was a sweeping combination of MSI and CWI. The sweeping parameters were defined as follows: the sweeping step should be less than or equal to $1 / T_{d}$, the sweeping frequency range width should be grater or equal to $1 \mathrm{kHz}$.

The MTLL should be shorter thanthe sweeping dwell time at each sweep frequency and the sweeping speed should be adjusted to guarantee that the interference frequency returns to its influence zone for time less than the reacquisition time.

\section{REFERENCES}

[1] M. J. Rezaei, et al., "New GPS anti-jamming system based on multiple short-time Fourier transform," IET Radar, Sonar and Navigation, vol. 10 pp. 807-815, 2016.

[2] R. Onrubia, et al., "Assessment of DME/TACAN RFI mitigation techniques in GNSS-R," in 2016 IEEE International Geoscience and Remote Sensing Symposium (IGARSS), 2016, pp. 4811-4814.

[3] Y. R. Chien, "Design of GPS Anti-Jamming Systems Using Adaptive Notch Filters," IEEE Systems Journal, vol. 9, pp. 451-460, 2015.

[4] Z. Xu, et al., "Weak interference direction of arrival estimation in the GPS L1 frequency band," in 2015 IEEE International Conference on Acoustics, Speech and Signal Processing (ICASSP), 2015, pp. 2649-2653.

[5] B. Zhang, et al., "Robust anti-jamming method for high dynamic global positioning system receiver," IET Signal Processing, vol. 10 , pp. 342-350, 2016.

[6] M. K. Bek, et al., "Classification and Mathematical Expression of Different Interference Signals on a GPS Receiver," Navigation, vol. 62 , pp. 23-37, 2015

[7] E. M. Shaheen and S. A. Elgamel, "Mathematical Analyses of the GPS Receiver Interference Tolerance and Mean Time to Loss Lock," Defence Technology,vol. 15, issue: 3, pp.440-449, 2019.

[8] P. W. Ward, "The Natural Measurements of a GPS Receiver," The Institute of Navigation, 51st Annual Meeting, Colorado Springs, CO, June 5-7, 1995.

[9] G. Gerten, "Protecting the Global Positioning System," IEEE Aerospace and Electronic Systems Magazine, vol 20, pp. 3-8, 2005

[10] B. W. Parkinson and J. J. Spilker, Eds., Global Positioning System: Theory and Applications. Washington DC: American Institute of Aeronautics and Astronautics, Inc, 1996.

[11] A. Jafarnia, et al., "Detection and Mitigation of Spoofing Attacks on a Vector-Based Tracking GPS Receiver," Institute of Navigation, Long Beach, CA, Jan 30 - Feb 1, 2012.

[12] S. v. Leeuwen, "Electromagnetic Interference on Low Cost GPS Receivers " National Aerospace Laboratory NLR 2008.

[13] C. J. Hegarty, "Suppression of Pulsed Interference Through Blanking," The International Association of Institutes of Navigation 25th World Congress/The Institute of Navigation 56th Annual Meeting, San Diego, CA, June 2000.

[14] C. Hegarty, A. J. Van Dierendonck, D. Bobyn, M. Tran, T. Kim, and J. Grabowski, "Suppression of Pulsed Interference through Blanking," ION Annual Meeting 26-28 June, San Diego, CA, pp. 399-408, U.S. Institute of Navigation, Fairfax, VA 2000.

[15] T. Lin, A. Broumandan and J. Nielsen, "Robust Beamforming for GNSS Synthetic Antenna Arrays," ION GNSS 2009, Session A5, 22-25, Savannah, GA, September 2009.

[16] A. M. M. Kamel, "Context Aware High Dynamics GNSS-INS forInterference Mitigation " PHD, Department of Geomatics Engineering, UNIVERSITY OF CALGARY, 2011.

[17] E. D. Kaplan and C. J. Hegarty, Eds., Understanding GPS Principles and Applications. Artech House, 2006.

[18] M. K. Bek, et al., "Mathematical analyses of pulse interference signal on post-correlation carrier-to-noise ratio for the global positioning system receivers," IET Radar, Sonar and Navigation, vol. 9, pp. 266-275, 2015. [19] J. Bao and Y.Tsui, Eds., Fundamentals of Global Positioning System Receivers: A Software Approach. A JOHN WILEY and SONS, INC, 2005.

[20] M. K. Bek, et al.,"Analysis ofglobal positioning system acquisition process in the presence of interference," IET Radar, Sonar and Navigation, vol. 10 , issue 5 , pp. $850-861,2016$ 